\title{
Quasi-static and dynamic in vitro mechanical response of 3D printed scaffolds with tailored pore size and architectures
}

DOI:

10.1016/j.msec.2018.11.019

\section{Document Version}

Accepted author manuscript

Link to publication record in Manchester Research Explorer

Citation for published version (APA):

Rotbaum, Y., Puiu, C., Rittel, D., \& Domingos, M. (2018). Quasi-static and dynamic in vitro mechanical response of $3 \mathrm{D}$ printed scaffolds with tailored pore size and architectures. Materials Science and Engineering: $C$. https://doi.org/10.1016/j.msec.2018.11.019

Published in:

Materials Science and Engineering: C

\section{Citing this paper}

Please note that where the full-text provided on Manchester Research Explorer is the Author Accepted Manuscript or Proof version this may differ from the final Published version. If citing, it is advised that you check and use the publisher's definitive version.

\section{General rights}

Copyright and moral rights for the publications made accessible in the Research Explorer are retained by the authors and/or other copyright owners and it is a condition of accessing publications that users recognise and abide by the legal requirements associated with these rights.

\section{Takedown policy}

If you believe that this document breaches copyright please refer to the University of Manchester's Takedown Procedures [http://man.ac.uk/04Y6Bo] or contact uml.scholarlycommunications@manchester.ac.uk providing relevant details, so we can investigate your claim.

\section{OPEN ACCESS}


Accepted Manuscript

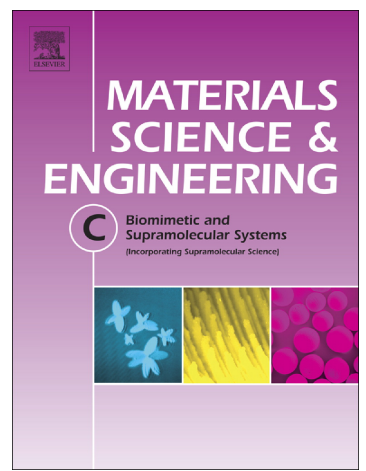

Quasi-static and dynamic in vitro mechanical response of 3D printed scaffolds with tailored pore size and architectures

Y. Rotbaum, C. Puiu, D. Rittel, M. Domingos

PII: $\quad$ S0928-4931(18)32665-1

DOI: $\quad$ https://doi.org/10.1016/j.msec.2018.11.019

Reference: $\quad$ MSC 9039

To appear in: $\quad$ Materials Science \& Engineering $C$

Received date: $\quad 3$ September 2018

Revised date: $\quad 22$ October 2018

Accepted date: $\quad 15$ November 2018

Please cite this article as: Y. Rotbaum, C. Puiu, D. Rittel, M. Domingos, Quasi-static and dynamic in vitro mechanical response of 3D printed scaffolds with tailored pore size and architectures. Msc (2018), https://doi.org/10.1016/j.msec.2018.11.019

This is a PDF file of an unedited manuscript that has been accepted for publication. As a service to our customers we are providing this early version of the manuscript. The manuscript will undergo copyediting, typesetting, and review of the resulting proof before it is published in its final form. Please note that during the production process errors may be discovered which could affect the content, and all legal disclaimers that apply to the journal pertain. 
Quasi-static and dynamic in vitro mechanical response of 3D printed scaffolds with tailored pore size and architectures

Y. Rotbaum ${ }^{1}$, C. Puiu ${ }^{2}$, D. Rittel ${ }^{1}$, M.Domingos ${ }^{2, *}$

${ }^{1}$ Faculty of Mechanical Engineering, Technion, 32000, Haifa, ISRAEL

${ }^{2}$ School of Mechanical, Aerospace and Civil Engineering, University of Manchester, UK

"Corresponding Author: Marco Domingos. Tel: (+44) 1613064889. Email: marco.domingos@manchester.ac.uk 


\section{Abstract}

Scaffold-based Tissue Engineering represents the most promising approach for the regeneration of load bearing skeletal tissues, in particular bone and cartilage. Scaffolds play major role in this process by providing a physical template for cells to adhere and proliferate whilst ensuring an adequate biomechanical support at the defect site. Whereas the quasi static mechanical properties of porous polymeric scaffolds are well documented, the response of these constructs under high strain compressive strains remain poorly understood. Therefore, this study investigates, for the first time, the influence of pore size and geometry on the mechanical behaviour of Polycaprolactone (PCL) scaffolds under quasi static and dynamic conditions. 3D printed scaffolds with varied pore sizes and geometries were obtained using different filament distances (FD) and lay-down patterns, respectively. In particular, by fixing the lay-down pattern at $0 / 90^{\circ}$ and varying the FD between 480 $980 \mu \mathrm{m}$ it was possible to generate scaffolds with square pores with dimensions in the range of 150$650 \mu \mathrm{m}$ and porosities of $59-79 \%$. On the other hand, quadrangular, hexagonal, triangular and complex pore geometries with constant porosity (approx. 70\%) were obtained at a fixed FD of 680 $\mu \mathrm{m}$ and imposing four different lay-down patterns of 0/90, 0/60/120, 0/45/90/135 and 0/30/60/90/120/150 ${ }^{\circ}$, respectively. The mechanical response of printed scaffolds was assessed under two different compression loading regimes spanning five distinct strain rates, from $10^{-2}$ to $2000 \mathrm{~s}^{-1}$, using two different apparatus: a conventional screw-driven testing machine (Instron 4483) and a Split Hopkinson pressure bar (SHPB) equipped with a set of A201 Flexi-forceTM (FF) force sensors and a pulse shaper. Our results show that the mechanical properties of PCL scaffolds are not strain rate sensitive between 1300 to $2000 \mathrm{~s}^{-1}$ and these strongly depend on the pore size (porosity) rather than pore geometry. Those findings are extremely relevant for the engineering of bone tissue scaffolds with enhanced mechanical stability by providing new data describing the mechanical response of these constructs at high strain rates as well as the at the transition between quasi static and dynamic regimes.

Keywords: 3D Bioprinting, scaffolds, mechanical response, quasi static, dynamic, high strain rate 


\section{Introduction}

The recent advances in materials science, stem cell biology and 3D Bioprinting have prompted the development of novel Tissue Engineering (TE) therapeutics for the regeneration of damaged tissues and organs. Particular attention has been dedicated to the use of "top-down" approaches where 3D scaffolds, cells and bioactive molecules are combined with the aim of recreating the native extracellular matrix (ECM) environment and promoting the guided neo-tissue ingrowth [1]. Despite the possibility to use this strategy for soft tissue engineering, it is the regeneration of load bearing tissues, such as bone and cartilage that keeps attracting the interest of different research groups worldwide [2-5]. The scaffold is instrumental in this approach as it provides a temporary template for cells to adhere, proliferate and differentiate, while ensuring an optimal mechanical stability at the site of implantation [6]. The orchestrated process by which cells remodel and deposit the ECM requires the scaffold to fulfil a wide range of chemical, physical and biological requirements, including appropriate pore size/geometry, porosity, biodegradability, biocompatibility, stiffness, easy processability and sterilization [7]. Moreover, reproducibility is highly desirable for the systematic in vitro evaluation of cell-cell or cell-matrix interactions. The paradigm shift in healthcare towards personalized medicine further reinforces the need to develop patient-customized scaffolds with tailored biochemical features for individualized treatment. From a manufacturing view point, 3D Bioprinting techniques, comprising laser-assisted, jetting and extrusion-based systems, offer a route to produce such constructs by providing engineers with the precise control over the spatial distribution/positioning of multiple cells and materials [8]. These computercontrolled techniques operate in a layer-by-layer fashion and can be combined with Computer Aided Design (CAD) or medical imaging to produce highly accurate patient-specific implants [9]. The flexibility, low cost and ease of operation typical of extrusion-based processes has promoted its widespread application in scaffold manufacturing for TE [10]. Additionally, the systems can be easily adapted to operate with a large range of materials spanning from synthetic polymers to organic-inorganic composites [11,12]. As extrusion-based bioprinting systems innovate, driven by progresses at the software and hardware levels, researchers continue to improve on the biomimetic design of scaffolds as a mean to achieve greater control over the tissue regeneration process. This encompasses the ability to tailor the biological and mechanical response of a scaffold through the manipulation of its internal architectural features, namely pore size and geometry. In a previous study, our group has shown that 3D printed Polycaprolactone (PCL) scaffolds with increased pore size (from 250 to $450 \mu \mathrm{m}$ ) and more regular geometries (quadrangular) favour the in vitro viability and proliferation of human Mesenchymal Stem Cells (hMSC's) [13]. We have also demonstrated that scaffolds with smaller dimensions $(250 \mu \mathrm{m})$ and regular geometries improved the mechanical 
response under static compression loading. The relatively simple tunability of geometrical/dimensional features offered by extrusion-based printers allied with the processability and biocompatiblity of PCL has prompted further studies on the generation of 3D scaffolds for in vivo bone regeneration. In a recent study, Caetano et al. observed that $3 \mathrm{D}$ printed PCL scaffolds seeded with Adipose Derived Stem Cells (ADSC's) and implanted in a rat calvarial model supported cell colonization whilst exhibiting $22 \%$ more bone formation than natural bone healing [14]. Despite these promising findings and the relatively well known mechanical behaviour of 3D bioextruded PCL scaffolds under quasi-static and cyclic loading, the behaviour of these constructs at high strain rates (impact) remains poorly understood [13,15-18]. This is of paramount importance for the dynamic mechano-regulated process by which osteoblasts and osteocytes deposit and resorbs bone tissue. This osteogenic process is well documented in the literature and comprises important parameters such as the load frequency, strain rate, peak strain, frequency and number of cycles/duration [19,20]. Going beyond those low intensity cyclic mechanical events, one may wonder about the behaviour of a scaffold when subjected to impact loading, of the kind encountered physically on a daily basis, e.g. during sports practice[20]. Such loading regimes and the mechanical response of bone have been investigated over a broad range of strain rates in order to better understand possible failure mechanisms [21-23]. Interestingly, these experimental studies, performed at high strain rates, have confirmed that mechanical behaviour is strain rate dependent and therefore needed to be taken into account when designing musculoskeletal devices [21-25]. Moreover, current clinical procedures are evolving towards earlier and faster mechanical loading of bone implants after surgery in order to promote enhanced tissue remodelling, reduce recovery time and improve patient comfort [26]. As such, developing a better understanding of the effect of different loading regimes on the dynamic mechanical performance of TE constructs becomes critical for the optimization of the scaffold's design thus ensuring an optimal long term biomechanical stability at the defect site, while shedding additional light on its mechanical resilience at high loading rates.

This study presents, for the first time, the quasi static and dynamic mechanical response of 3D bioprinted Polycaprolactone (PCL) scaffolds under compression at high strain rates, whose pore size and geometry are systematically varied.

\section{Materials and Methods}

\subsection{Materials}

Poly(E-caprolactone) (PCL) (CAPA 6500, Mw = 50.000 Da, Perstorp Caprolactones - Cheshire, United Kingdom) in the form of pellets was employed as received for the fabrication of threedimensional (3D) scaffolds. 


\subsection{Scaffold design and fabrication}

3D PCL scaffolds were produced using a commercial extrusion-based bioprinter (regenHU, Switzerland) equipped with a high temperature printing head and a nozzle of $330 \mu \mathrm{m}$. A detailed description of the equipment as well as the flowchart information required for the generation of TE scaffolds can be found elsewhere [27]. Independently of pore size and geometry and using the BioCAD software, rectangular prisms measuring 30 (Length) x 30 (Width) x 5 (Height) mm were initially designed. Afterwards, imposing a fixed lay-down pattern of $0 / 90^{\circ}$ and varying the filament distance (FD) between $480 \mu \mathrm{m}$ and $980 \mu \mathrm{m}$ it was possible to obtain scaffolds with square pores and different dimensions (Figure 1). Pore geometry variations were obtained by imposing a constant filament distance (FD) of $680 \mu \mathrm{m}$ and adopting four different lay-down patterns or angles of $0 / 30 / 60 / 90 / 120 / 150^{\circ}, 0 / 45 / 90 / 135^{\circ}, 0 / 60 / 120^{\circ}$ and $0 / 90^{\circ}$ (Figure 2). Process parameters were set according to previous works and can be found elsewhere [27]. After being printed, the scaffolds were removed from the building platform and cut into smaller specimens with adequate dimensions for further analyses.

a)

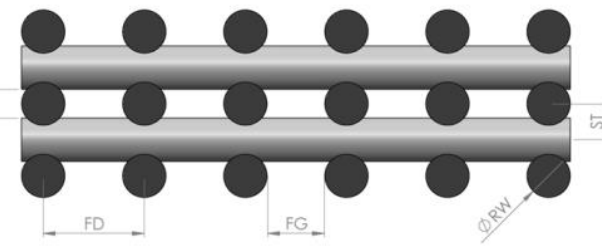

b)

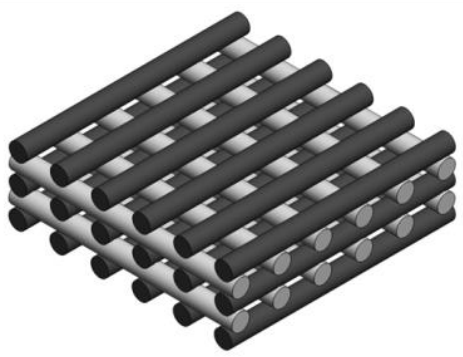

Figure 1: 2D (XY plane) and 3D schematic representation of a printed construct characterized by a $0^{\circ} / 90^{\circ}$ lay-down pattern. Symbols are denoted as RW: road width or filament diameter; FG: filament gap; ST: slice thickness; LG: layer gap; FD: filament distance (centre to centre).

a)

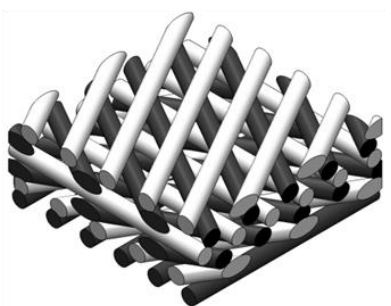

b)

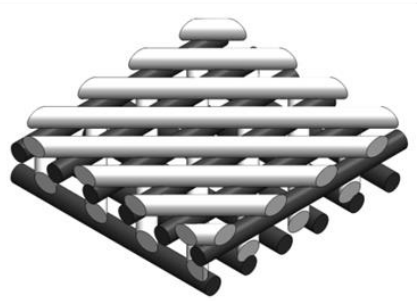

c)

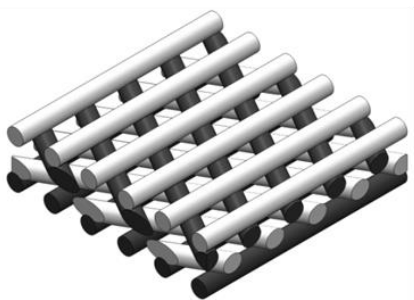

d)

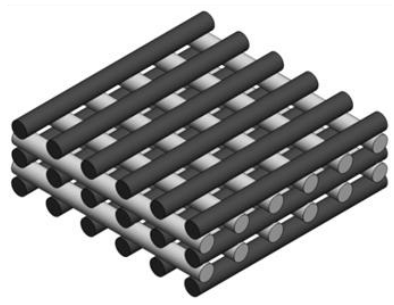

Figure 2: 3D CAD models of printed scaffolds characterized by constant FD and varied lay-down pattern. a) $0 / 30 / 60 / 90 / 120 / 150^{\circ}$; b) $0 / 45 / 90 / 135^{\circ}$; c) $0 / 60 / 120^{\circ}$; c) $0 / 90^{\circ}$ 


\subsection{Scaffold's characterization}

\subsubsection{Morphological analysis}

Morphological features of 3D printed scaffolds, such as pore size/geometry and filament diameters were assessed through Scanning Electron Microscopy (SEM) using a FEI Quanta 200. Top and cross-section micrographs were obtained under high vacuum conditions, voltage of $20.0 \mathrm{kV}$ and pressure of $3.2 \times 10^{-5}$ Torr.

\subsubsection{Porosity}

The porosity of 3D printed scaffolds was measured analytically employing the following methodology: (1) measuring the weight and volume of each sample, (2) calculating the apparent density of the PCL scaffolds, (3) applying the formula reported below:

$$
\text { Porosity }=\left(1-\frac{\rho^{*}}{\rho_{\text {sub }}}\right) \times 100
$$

Where $\rho^{*}$ is the apparent density of the scaffold and $\rho_{\text {sub }}$ is the density of the PCL material $\left(\rho_{\text {sub }}=1\right.$. $145 \mathrm{~g} / \mathrm{cm}^{3}$ ). Five samples were measured for each group of parameters.

\subsection{Mechanical testing}

\subsubsection{Quasi-static testing}

Uniaxial quasi-static compression experiments were conducted using a screw-driven testing machine (Instron 4483), under displacement control, with a prescribed crosshead to a nominal strain rate of $10^{-2} \mathrm{~s}^{-1}$. For high resolution measurement, the force (F) was measured using a $500 \mathrm{~N}$ load-cell and the uniaxial displacement was measured using LE-05, EIR, laser extensometer. The measured load-displacement curves were reduced into engineering stress-strain curves, where the engineering stress $\left(\sigma_{\text {eng }}\right)$ was calculated as the applied load divided by the original cross section area $\left(\mathrm{A}_{0}\right)$ with respect to the scaffold's porosity $(\phi)$.

$$
\sigma_{\text {eng }}=\frac{F}{A_{0}(1-\phi)}
$$

Since the specimen's cross section area doesn't change much during the test, the engineering strain is a reliable measure. The engineering strain $\left(\varepsilon_{\text {eng }}\right)$ was calculated as:

$$
\varepsilon_{\text {eng }}=\frac{\Delta L}{L_{0}}
$$

Where $\mathrm{L}_{0}$ is the original specimen height and $\Delta \mathrm{L}$ is the measured displacement. One should note that, all the experiments were terminated when the load-cell reading reached $500(\mathrm{~N})$, which is the load-cell limit. 
For the quasi-static experiments, square parallelepiped samples with a nominal cross section of $7 X 7 \mathrm{~mm}$ and $5 \mathrm{~mm}$ height were cut from the main scaffold.

\subsubsection{Dynamic testing}

The dynamic compression experiments were performed using a conventional $12.7 \mathrm{~mm}$ diameter Split Hopkinson pressure bar (SHPB), made of 7075-T6 aluminum alloy bars, which were loaded at the far end of the incident bar with a projectile made of the same material [28]. In conventional SHPB setups, the forces and the displacements measurement is usually done using strain gauges attached to the Hopkinson bars. The strain gauges record the incident, reflected and transmitted stress waves, $\varepsilon_{\text {inc,, }} \varepsilon_{\text {ref }}$ and $\varepsilon_{\text {tra }}$, respectively, which are then converted into stress and strain pulses[29].

Experimenting with soft materials whose mechanical impedance is significantly lower than that of the Hopkinson bars, poses a significant experimental challenge [30-35]. While in a conventional experiment the forces' amplitude lies in the range of $15,000-40,000 \mathrm{~N}$, in our experimental setup the measured forces were in the range of $60-400 \mathrm{~N}$. The low force amplitude causes difficulties in the force measurement and in achieving dynamic force equilibrium, which is essential for converting the strain gauges signals into stress-strain curves [35,36]. In order to overcome this problem, a set of A201 Flexi-force ${ }^{\mathrm{TM}}(\mathrm{FF})$ force sensors were cemented on the edges of the Hopkinson's bar, so that the interfacial forces were measured directly, and not through gauge signal analysis [37]. In addition a pulse shaper, consisting of soft paper mixed with a carefully measured amount of molybdenum disulfide grease, was inserted between the striker and the incident bar [30,36]. The pulse shaper is used to increase the rise time of the loading pulse, thereby improving the specimen's equilibrium due to reduced accelerations [38].

For the dynamic experiments, square parallelepiped samples with a nominal cross section of $5 \times 5 \mathrm{~mm}$ and $5 \mathrm{~mm}$ height were cut from the main scaffold. The given dimensions insured that the specimens would always be well contained within the sensing area of the FF sensor.

\section{Results and Discussion}

\subsection{Morphological analysis}

Scanning electron microscopy performed on 3D printed scaffolds show that, independently of the FD and lay-down pattern, all constructs present a well-defined internal geometry with open pores and good adhesion between adjacent layers. As illustrated in Figure 3, PCL scaffolds produced with a single lay-down pattern of $0^{\circ} / 90^{\circ}$ and varied FD (between $480 \mu \mathrm{m}$ and $980 \mu \mathrm{m}$ ) resulted in constructs with square interconnected pores of regular dimensions (FG) in the range of $148 \mu \mathrm{m}$ $652 \mu \mathrm{m}$. 

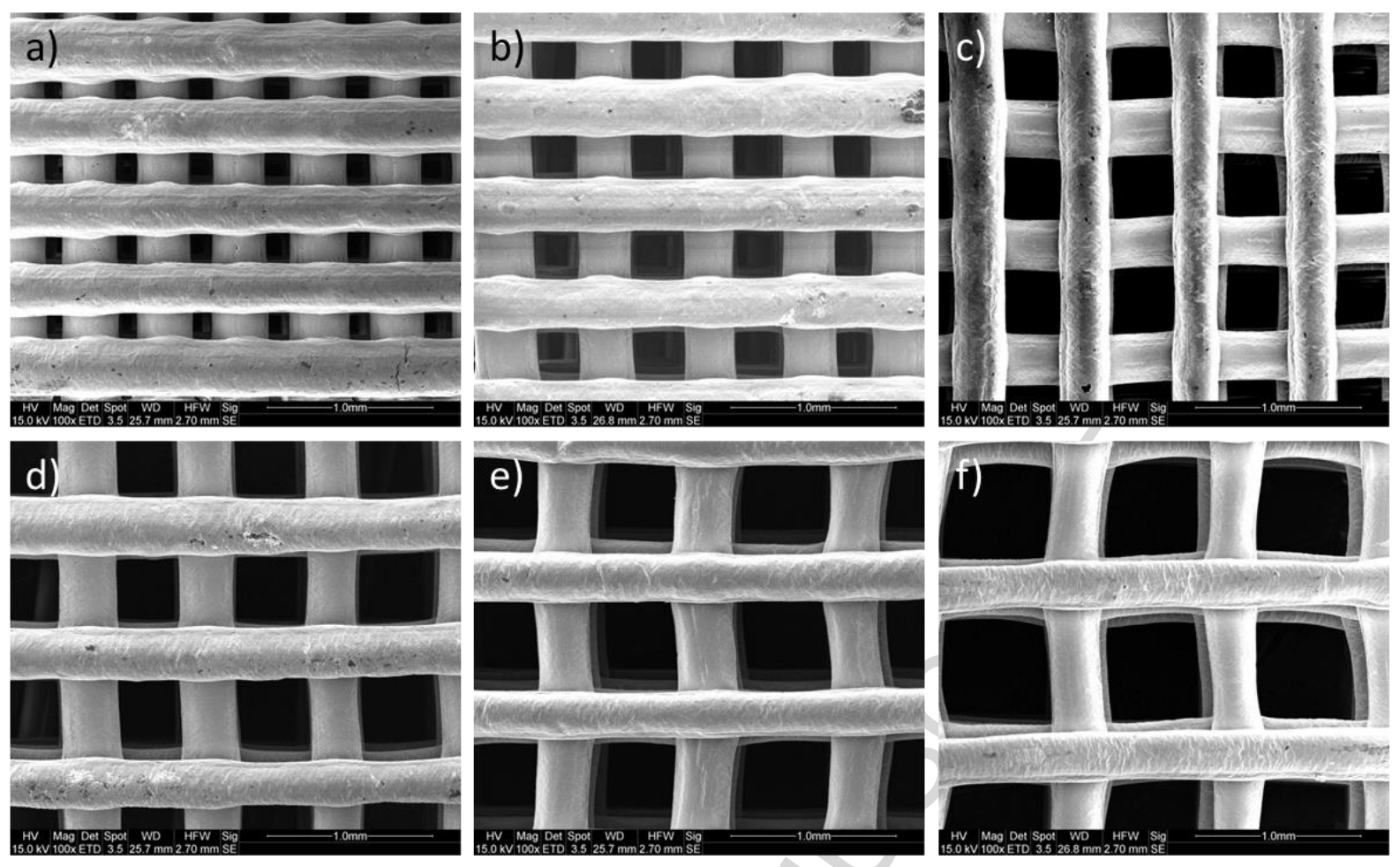

Figure 3: Top SEM micrographs of PCL scaffolds with constant lay-down pattern of $0 / 90^{\circ}$ and different FD values. a) FD = $480 \mu \mathrm{m}$; b) FD = $580 \mu \mathrm{m}$; c) FD = $680 \mu \mathrm{m}$; d) FD = $780 \mu \mathrm{m}$; e) FD = $880 \mu \mathrm{m} ; \mathrm{f}) \mathrm{FD}=980 \mu \mathrm{m}$.

A significant consistency between the theoretical values and experimental measurements for RW, FG and LG were obtained thus confirming the accuracy and reproducibility of the 3D printing system employed in the production of the PCL scaffolds (Table 1). Through the systematic variation of FD it was also possible to generate 3D constructs with different levels of porosity ranging between $59 \%$ and $79 \%$ (Table 1 ).

Table 1: Experimental measurements of 3D printed scaffolds with fixed lay down pattern of $0 / 90^{\circ}$ and varied FD.

\begin{tabular}{lllll}
\hline FD $(\boldsymbol{\mu m})$ & RW $(\boldsymbol{\mu m})$ & FG $(\boldsymbol{\mu m})$ & LG $(\boldsymbol{\mu m})$ & Porosity $(\boldsymbol{\%})$ \\
\hline $\mathbf{4 8 0}$ & $329 \pm 6$ & $148 \pm 5$ & $194 \pm 10$ & 59 \\
$\mathbf{5 8 0}$ & $332 \pm 7$ & $251 \pm 9$ & $196 \pm 7$ & 66 \\
$\mathbf{6 8 0}$ & $329 \pm 7$ & $347 \pm 9$ & $200 \pm 6$ & 70 \\
$\mathbf{7 8 0}$ & $329 \pm 9$ & $450 \pm 8$ & $196 \pm 3$ & 74 \\
$\mathbf{8 8 0}$ & $328 \pm 5$ & $552 \pm 8$ & $194 \pm 11$ & 77 \\
$\mathbf{9 8 0}$ & $326 \pm 8$ & $652 \pm 5$ & $192 \pm 6$ & 79 \\
\hline
\end{tabular}


Defining a constant FD of $680 \mu \mathrm{m}$ and imposing four different lay-down patterns of $0 / 30 / 60 / 90 / 120 / 150^{\circ}, 0 / 45 / 90 / 135^{\circ}, 0 / 60 / 120^{\circ}$ and $0 / 90^{\circ}$, it was possible to produce $3 \mathrm{D}$ scaffolds with complex polygonal, triangular, hexagonal and quadrangular pore geometries, respectively (Figure 4).
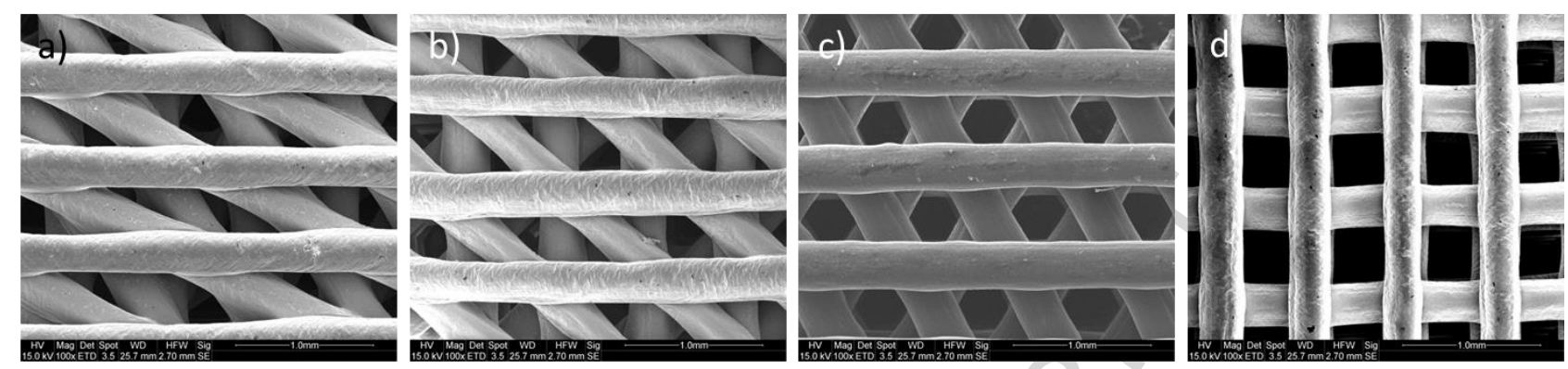

Figure 4: Top SEM micrographs of PCL scaffolds with constant FD of $680 \mu \mathrm{m}$ and different laydown patterns. a) $0 / 30 / 60 / 90 / 120 / 150^{\circ}$; b) $0 / 45 / 90 / 135^{\circ}$; c) $0 / 60 / 120^{\circ}$; d) $0 / 90^{\circ}$.

As reported in Table 2, the variation of lay-down pattern from $0 / 30 / 60 / 90 / 120 / 150^{\circ}$ to $0 / 90^{\circ}$ resulted in constructs with great consistency between pre-defined and experimentally measured geometrical features. Porosity levels, calculated based on equation 1, were not observed to change significantly $(67 \%-70 \%)$ due to the constant FD imposed during the printing process.

Table 2: Experimental measurements of 3D printed scaffolds with fixed FD of $680 \mu \mathrm{m}$ and varied lay down patterns.

\begin{tabular}{lllll}
\hline Lay-down pattern $\left({ }^{\circ}\right)$ & RW $(\boldsymbol{\mu m})$ & FG $(\boldsymbol{\mu m})$ & LG $(\boldsymbol{\mu m})$ & Porosity $(\%)$ \\
\hline $\mathbf{0 / 3 0 / 6 0 / 9 0 / 1 2 0 / 1 5 0}$ & $319 \pm 6$ & $356 \pm 6$ & $1152 \pm 4$ & 67 \\
$\mathbf{0 / 4 5 / 9 0 / 1 3 5}$ & $322 \pm 7$ & $354 \pm 7$ & $719 \pm 5$ & 70 \\
$\mathbf{0 / 6 0 / 1 2 0}$ & $334 \pm 9$ & $348 \pm 4$ & $462 \pm 7$ & 70 \\
$\mathbf{0 / 9 0}$ & $329 \pm 7$ & $347 \pm 9$ & $200 \pm 6$ & 70 \\
\hline
\end{tabular}

\subsection{Mechanical response of the scaffolds}

\subsubsection{Quasi-static}

As mentioned above, the mechanical response to quasi-static loading is one of the most basic characteristics of a load baring component. The implanted scaffold should support diverse everyday activities like sitting, lying down and walking whilst ensuring adequate pore volumes for adherent cells to proliferate and differentiate. In those cases, the applied loading is essentially quasi-static. The quasi-static experiments are divided into two sections that investigate the mechanical response of the scaffolds under compression loading when: 1) the FD is varied and the 
lay-down pattern is fixed at $0 / 90^{\circ} ; 2$ ) the lay-down pattern (pore geometry) is varied and the FD is kept constant at $680 \mu \mathrm{m}$.

Typical engineering (nominal) stress-strain curves of the various scaffolds together with that of fully dense PCL are shown in Figure 5.

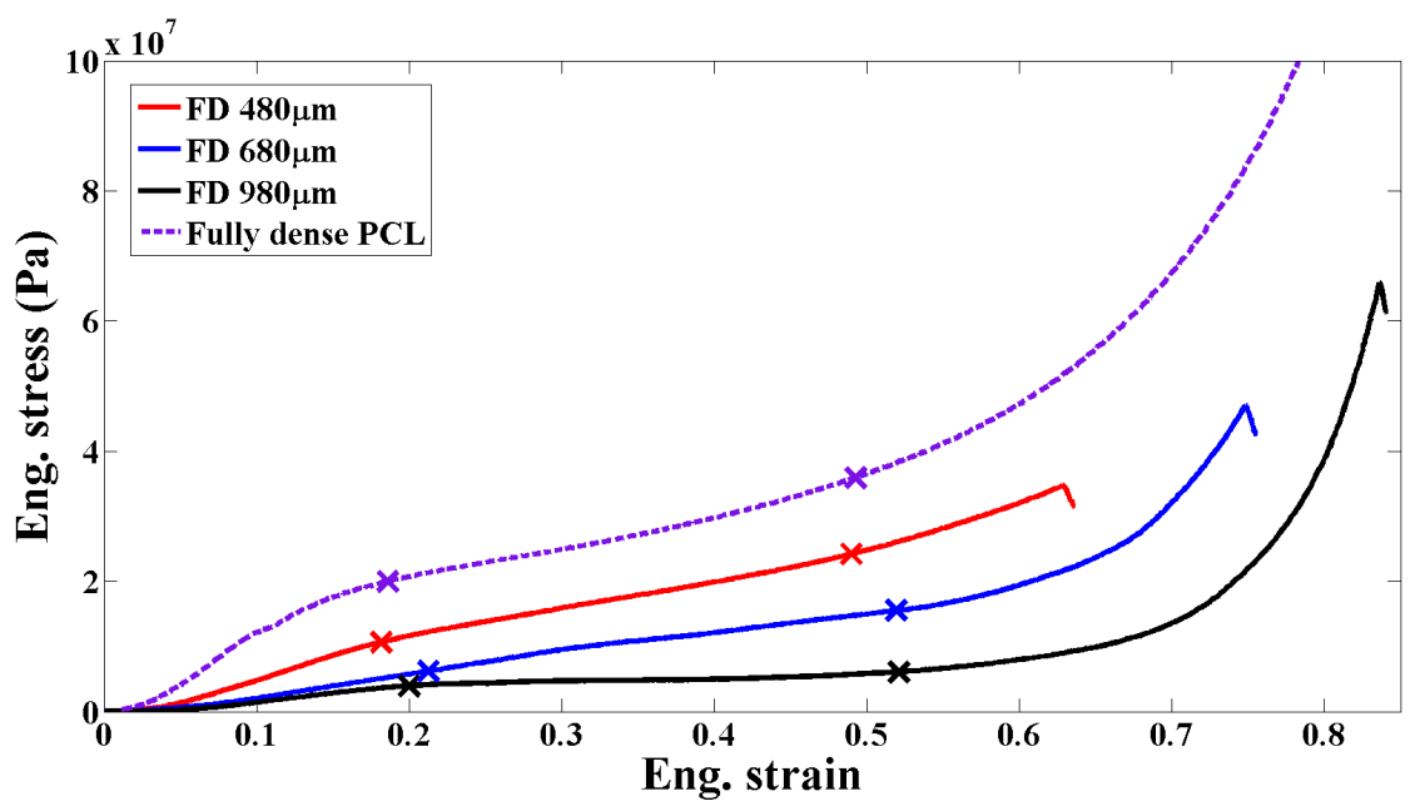

Figure 5. Typical quasi-static nominal stress-strain curves of $0 / 90^{\circ}$ scaffolds with FD of 480,680 and $980 \mu \mathrm{m}$, respectively, and fully dense PCL. The markings indicate the observed transition in the hardening phases. Note that none of the specimens actually fractured.

The engineering stress-strain curves plotted in Figure 5 show that, independently of the FD, all scaffolds display a mechanical response characterized by three main hardening phases: 1) The first hardening phase is related directly to the single filament ability to withstand physical load which extends up to an approximate strain of $0.2 ; 2$ ) The second hardening phase occurs at strains in the

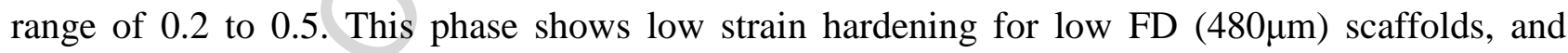
almost no strain hardening for large FD $(980 \mu \mathrm{m})$; 3) The third hardening phase starts when the gaps between the filaments are closed, so that densification proceeds bringing the material's response close to that of dense PCL. This is supported by previous observations where 3D printed scaffolds exhibit a mechanical behaviour under static compression similar to that of flexible foams [7,39]. This is particularly evident in the case of scaffolds with higher FD's $(980 \mu \mathrm{m})$ where the central region $(0.2<$ Eng. Strain $<0.5)$ is characterized by a stress plateau.

The observed differences in the mechanical responses of the scaffolds are directly related to the FD design. A possible explanation could be that upon sufficient compression of the filaments, especially for the cases where $\mathrm{LG} \cong \mathrm{FG}$, the lateral expansion of the filaments will act to reduce the 
FG down to a point where the filaments will contact each other, thereby stiffening the overall structure. This is even more evident for scaffolds with small values of FD $(480 \mu \mathrm{m})$ where the difference between the hardening phases becomes blurred. All the results pertaining to the $0 / 90^{\circ}$ tested scaffolds submitted to quasi-static loading at strain rate of $10^{-2} \mathrm{~s}^{-1}$ are shown in Figure 6. The results presented with respect to the actual cross section area.

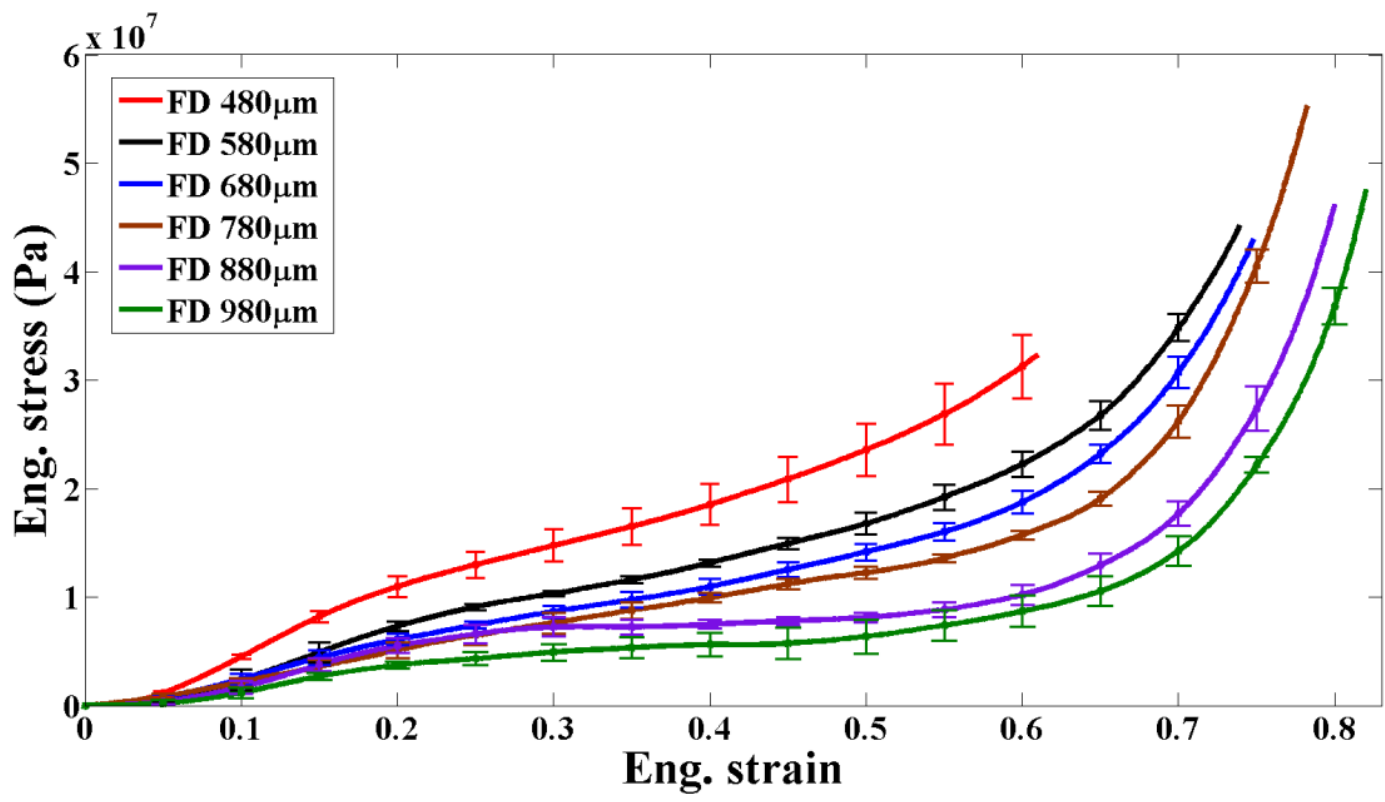

Figure 6. Average quasi-static engineering stress-strain curves of $0 / 90^{\circ}$ scaffolds with the calculated standard error bars.

As depicted in Figure 6, scaffolds with smaller pores sizes (lower values of FD and FG) exhibit higher flow stress compared to scaffolds with lager pore sizes. In addition, when measuring the mechanical response of soft materials, the small force amplitudes may lead to deviation in the measured flow stress, therefore the relatively small error bars indicate the quality and the reliability of the printing process [35]. The results reported in Figure 5 and 6 are in line with previous observations from other groups and show a direct dependence between the mechanical response of the scaffolds (at low strain rates) and their level of porosity $[13,16,39,40]$.

Finally in order to measure the influence of the lay-down pattern at a constant FD on the mechanical response, additional scaffolds with FD of $680 \mu \mathrm{m}$ and different printing angles were tested, namely $0 / 30 / 60 / 90 / 120 / 150^{\circ}, 0 / 45 / 90 / 135^{\circ}, 0 / 60 / 120^{\circ}$ and $0 / 90^{\circ}$. The measured stress-strain curves tested at nominal strain rate of $10^{-2} \mathrm{~s}^{-1}$ are shown in Figure 7. 


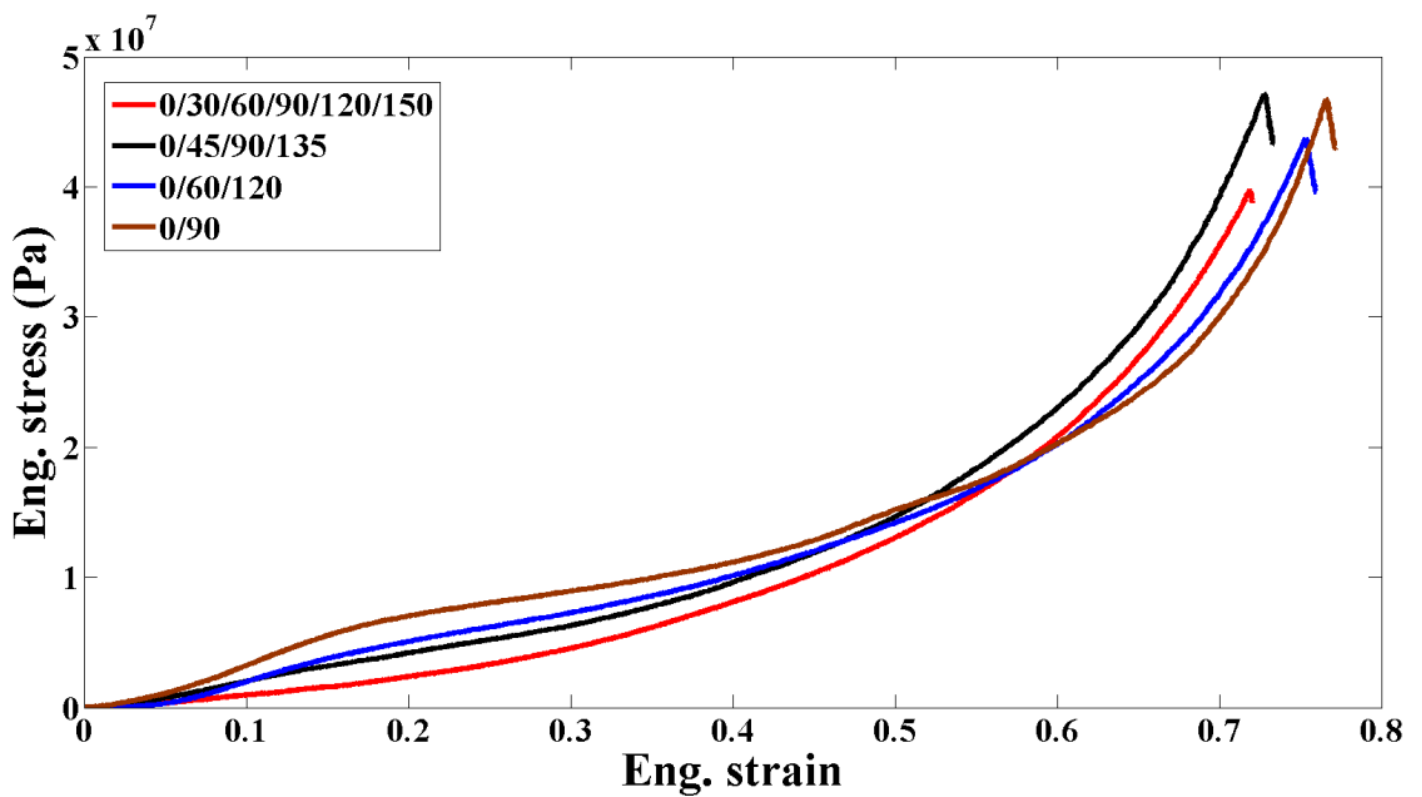

Figure 7. Average quasi-static engineering stress-strain curves of scaffolds with FD $680 \mu \mathrm{m}$ with different printing orientation. The flow stress is presented with standard error bars.

Figure 7 shows that scaffolds with different lay-down patterns exhibit a mechanical response that is rather similar to that of the $0 / 90^{\circ}$ scaffolds. The minor deviation observed for the $0 / 45 / 90 / 135^{\circ}$ scaffolds at strains higher than 0.5 , is due to shear deformation at the last part of the experiment. These results further support the observations reported in Figure 5 and 6 where the compressive strength of the constructs is mainly determined by the porosity level rather than the geometry of the pores (lay-down pattern). Due to this observation, the subsequent dynamic experiments focused only on the effect of FD in $0 / 90^{\circ}$ scaffolds. Moreover, and independently of the pore size or geometry, all 3D printed scaffolds display a compressive stress similar to that of human trabecular bone (4-12 MPa) thus confirming its potential in bone TE [41].

\subsubsection{Dynamic}

Although the field of high rate loading is overlooked in many cases, the characterization of the scaffolds response to impacts has a major importance in many aspects. High strain rate loading can change the scaffolds mechanical response in terms of hardening, recoverable vs. permanent damage and more. Similarly to the quasi-static load loading, high rate loading may also be experienced on a daily basis. Even more important, accidents like falling, bumping into a person on the street or even car accidents can deliver sharp shock loading into the body. Therefore a rigorous dynamic characterization is required to accurately predict the failure mechanisms of skeletal tissues and improve the design and modelling of artificial implants [42]. The dynamic experiments were carried out under compression and employing different strain rates ranging from 1300 to $2000 \mathrm{~s}^{-1}$. This range of strain has been previously employed in the mechanical characterization of human and 
bovine bones and can reveal whether the designed scaffolds are strain rate sensitive in the dynamic loading regime[21-23]. Characteristic flow curves of $0 / 90^{\circ}$ scaffolds with FD of $480 \mu \mathrm{m}$ at different strain rates are shown in Figure 8.

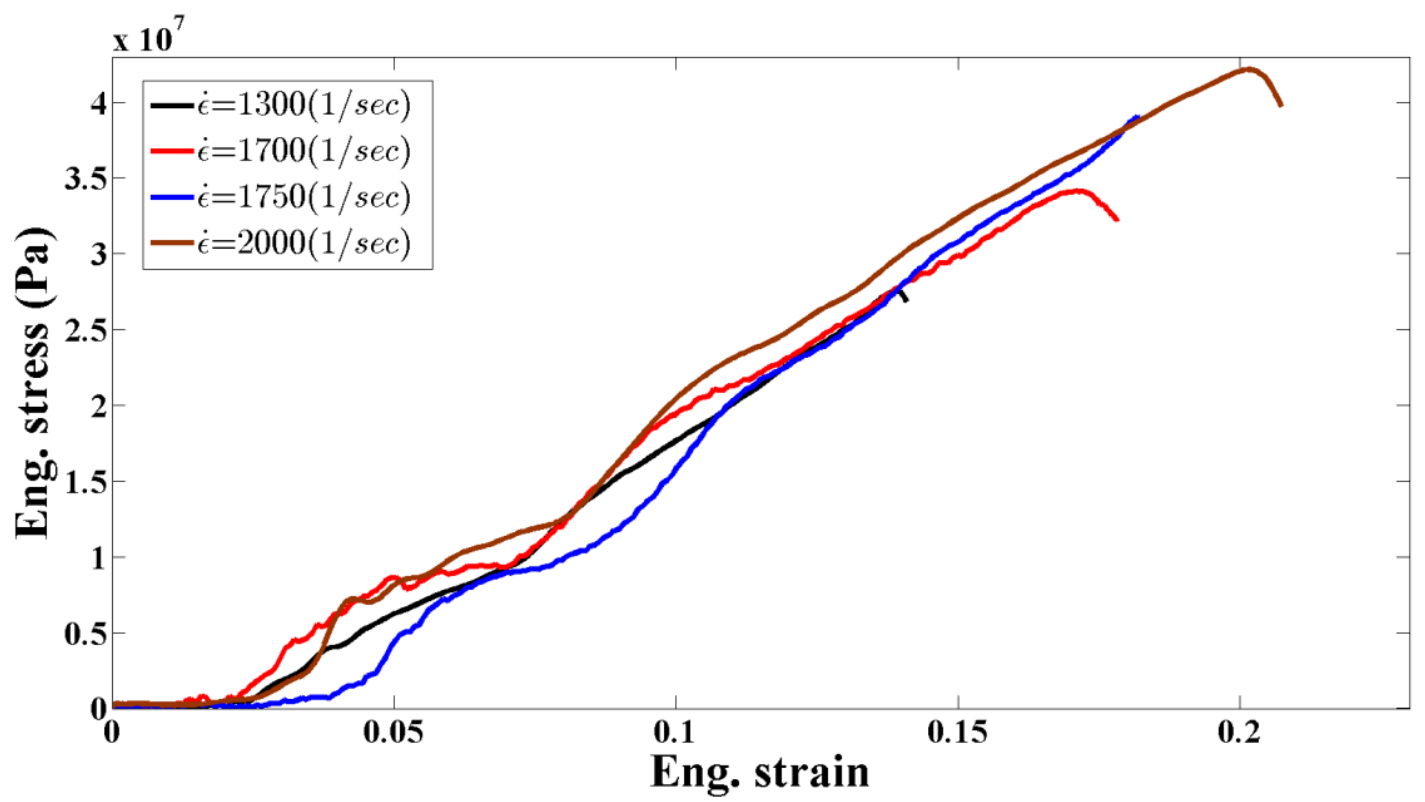

Figure 8. Typical dynamic nominal stress-strain curves of $0 / 90^{\circ}$ scaffolds with FD of $480 \mu \mathrm{m}$, at varied strain rates.

Figure 8 shows how the dynamic mechanical response of the scaffolds changes in respect to the static compression which was characterized by a three-phase hardening stress-strain curve. In this case the dynamic behaviour exhibits very small, almost no strain hardening up to a strain of $3 \%$, and from thereon, the scaffolds exhibit a constant rate of hardening. Moreover, within the range of employed strain rates, the scaffolds did not exhibit any significant strain rate sensitivity. This observation was common to all tested scaffolds independently of their FD and at fixed lay-down pattern of $0 / 90^{\circ}$ (Figure 9). These results appear to contradict previous findings from other researchers that suggest a dependence between the mechanical strength of human bone and the rate of applied strain [21]. However, it would be unreasonable at this point, to draw any comparisons between the two studies, especially because the range of applied strain rates is significantly different $\left(0.001\right.$ to $\left.1500 \mathrm{~s}^{-1}\right)$. 


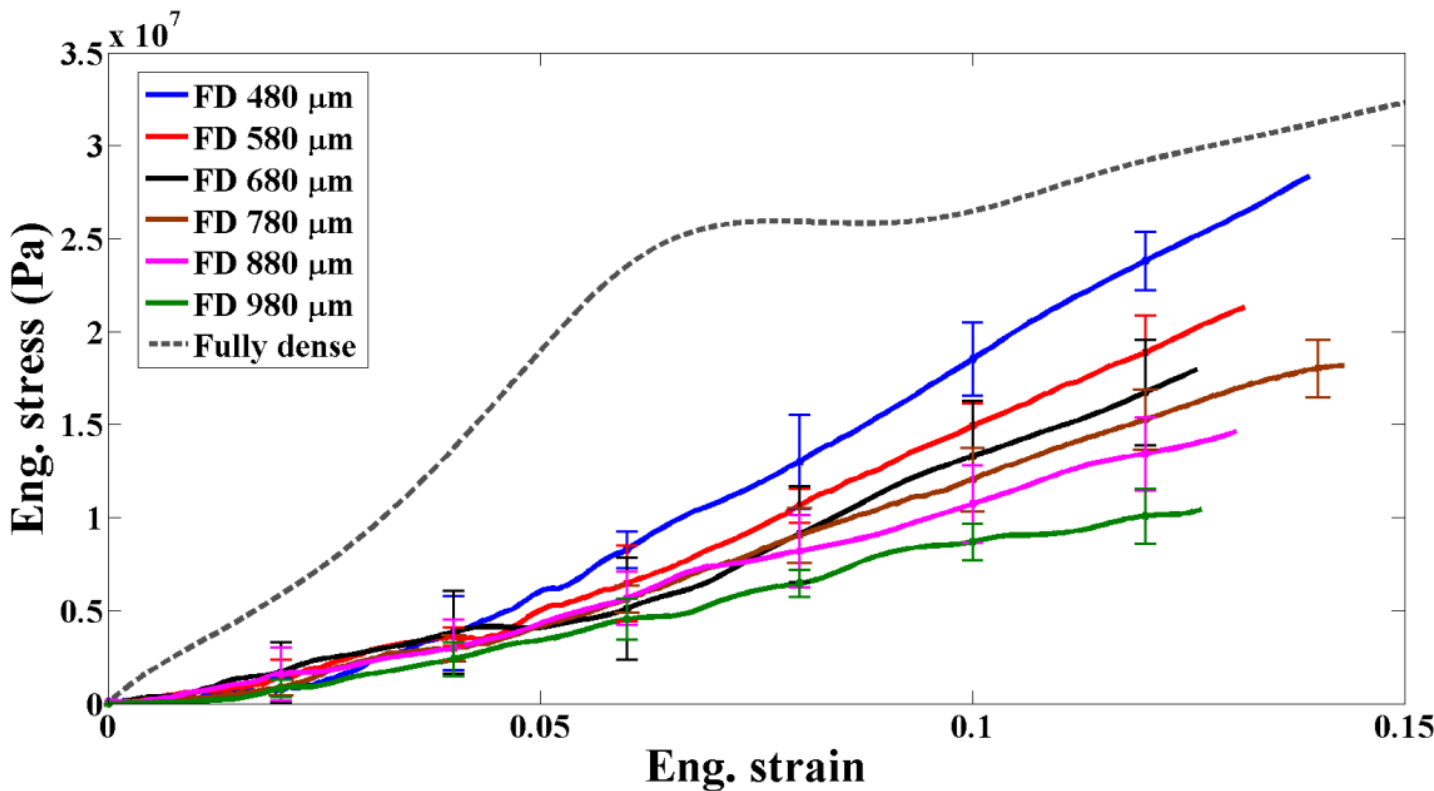

Figure 9. Average dynamic engineering stress-strain curves of $0 / 90^{\circ}$ scaffolds and fully dense PCL with the calculated standard error bars.

According to Figure 9, and similarly to the quasi static tests (Figure 7), scaffolds with smaller FD exhibit higher strain hardening in comparison to those with higher FD. However, the data indicate a much sharper increase of compressive stress under dynamic conditions in the strain interval between 0 and 0.1 . These findings are in accordance with others in the literature that suggest a strong correlation between the compressive stress and porosity (or volume fraction) of bone [24]. The experiments carried by Carter et al on the compressive response of bone further support our findings thus proposing the strength of the scaffolds to be a much stronger function of the porosity (or density) rather than the applied strain rate [43].

In order to further examine the strain rate sensitivity, a comparison between the quasi-static and dynamic response of scaffolds with $0 / 90^{\circ}$ lay-down pattern and FD of $480 \mu \mathrm{m}$ and $980 \mu \mathrm{m}$ is shown in Figure 10. Those scaffolds were selected for the comparison since under quasi-static loading they exhibit the strongest and the weakest flow stress, thereby providing bounds for the properties. 


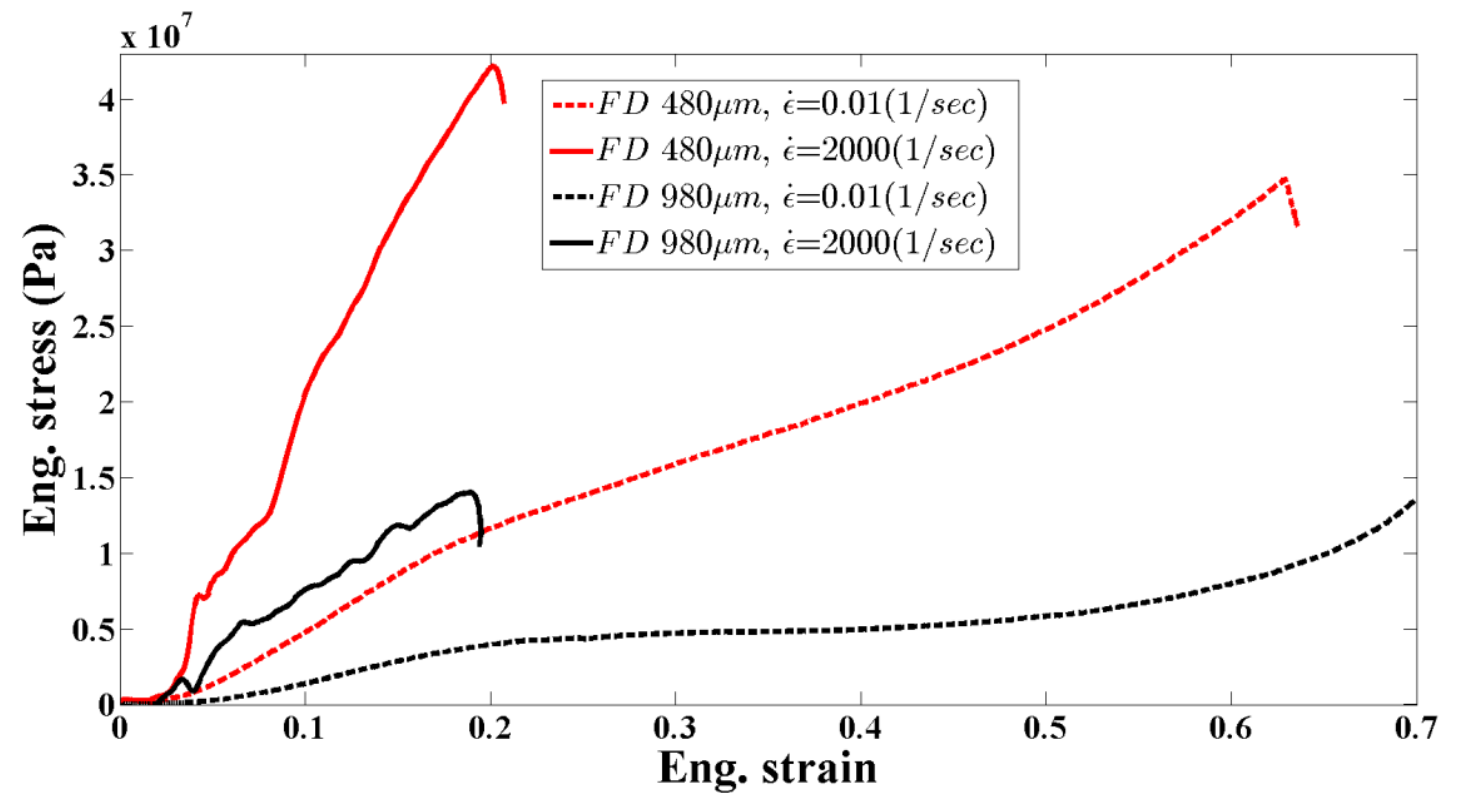

Figure 10. A comparison between scaffolds with FD 480 and $980 \mu \mathrm{m}$ submitted to quasi-static and dynamic loading.

Figure 10 clearly shows that the scaffolds are strain rate sensitive when shifting from static to dynamic loading. When these results are compared with those of the fully dense material (Figure 9), a pure material influence would most likely yield identical normalized stress strain curves. Since this is not the case, it appears that part of the strain rate sensitivity is related to the geometry of the scaffold and inertial effects in the dynamic regime. It's also worth mentioning that all scaffolds tested under dynamic conditions exhibit lower compressive stress compared to human bone with similar porosity (trabecular) [22]. This can be attributed to the fact that PCL scaffolds do not entirely mimic the two-phase composition of native bone, where the mineral phase (mainly composed of calcium phosphates) is responsible for the increased mechanical strength of the tissue.

\section{Conclusions}

The quasi static and dynamic compressive mechanical response of 3D printed PCL scaffolds with different pore sizes and geometries was experimentally evaluated in this work. As confirmed by the SEM micrographs, through the manipulation of design parameters (FD and lay-down pattern) it was possible to obtain highly accurate and reproducible 3D constructs characterized by a fully open network of interconnected pores. Imposing a single lay-down pattern of $0 / 90^{\circ}$ and varying the FD between $480 \mu \mathrm{m}$ and $980 \mu \mathrm{m}, 3 \mathrm{D}$ scaffolds were printed with pore sizes and porosities in the range of $148 \pm 5-652 \pm 5 \mu \mathrm{m}$ and 59-79\%, respectively. Quadrangular, hexagonal, triangular and complex pore geometries with constant porosity (approx. 70\%) were obtained at a fixed FD of $680 \mu \mathrm{m}$ and imposing four different lay-down patterns of 0/90, 0/60/120, 0/45/90/135 and 0/30/60/90/120/150 , 
respectively. The quasi static analyses revealed that, independently of the pore size and geometry, all scaffolds displayed a stress-strain curve characterized by a three-phase hardening mechanism. It was also possible to observe that the mechanical properties of PCL scaffolds are mainly determined by their pore size (FG) and that the compressive stress is enhanced by a lower porosity level. Dynamic tests at strain rates in the range of 1300 to $2000 \mathrm{~s}^{-1}$ performed using a SHPB apparatus showed that the mechanical response of 3D scaffolds printed with a constant lay-down pattern of $0 / 90^{\circ}$ and varied FD is not strain rate sensitive. This observation is valid for all tested strain rates independently of pore size or geometry except when the mechanical behaviour of 3D printed PCL scaffolds $(\mathrm{FD}=480 \mu \mathrm{m})$ is evaluated at $10^{-2}$ and $2000 \mathrm{~s}^{-1}$ strain rates. In this case, shifting from quasi static to dynamic conditions the scaffolds display evident strain rate sensitivity similar to that of human native bone. In conclusion, this study provides, for the first time, important information on the dynamic mechanical response of 3D printed PCL scaffolds under high compressive strain rates that can be used for the design and manufacturing of skeletal implants with enhanced biomechanical stability. Further work is already underway to investigate the behaviour of organic-inorganic composite (polymer/ceramic) 3D scaffolds at strain rates between 10 and $200 \mathrm{~s}^{-1}$ when immersed in Simulated Body Fluid (SBF). This will allow complementing the results obtained in this work whilst providing a better understanding on the effect of a bone-like mineral phase and SBF on the scaffold's response at low cycle loading regimes.

\section{References}

[1] S.M. Oliveira, R.L. Reis, J.F. Mano, Towards the design of 3D multiscale instructive tissue engineering constructs: Current approaches and trends, Biotechnol. Adv. 33 (2015) 842-855. doi:10.1016/J.BIOTECHADV.2015.05.007.

[2] W. Shi, M. Sun, X. Hu, B. Ren, J. Cheng, C. Li, X. Duan, X. Fu, J. Zhang, H. Chen, Y. Ao, Structurally and Functionally Optimized Silk-Fibroin-Gelatin Scaffold Using 3D Printing to Repair Cartilage Injury In Vitro and In Vivo, Adv. Mater. 29 (2017) 1701089. doi:10.1002/adma.201701089.

[3] M. Domingos, A. Gloria, J. Coelho, P. Bartolo, J. Ciurana, Three-dimensional printed bone scaffolds: The role of nano/micro-hydroxyapatite particles on the adhesion and differentiation of human mesenchymal stem cells, Proc. Inst. Mech. Eng. Part H J. Eng. Med. 231 (2017) 555-564. doi:10.1177/0954411916680236.

[4] E. Nyberg, A. Rindone, A. Dorafshar, W.L. Grayson, Comparison of 3D-Printed Poly- $\varepsilon-$ Caprolactone Scaffolds Functionalized with Tricalcium Phosphate, Hydroxyapatite, Bio-Oss, or Decellularized Bone Matrix, Tissue Eng. Part A. 23 (2017) 503-514. 
doi:10.1089/ten.tea.2016.0418.

[5] L. Roseti, V. Parisi, M. Petretta, C. Cavallo, G. Desando, I. Bartolotti, B. Grigolo, Scaffolds for Bone Tissue Engineering: State of the art and new perspectives, Mater. Sci. Eng. C. 78 (2017) 1246-1262. doi:10.1016/J.MSEC.2017.05.017.

[6] J. Henkel, M.A. Woodruff, D.R. Epari, R. Steck, V. Glatt, I.C. Dickinson, P.F.M. Choong, M.A. Schuetz, D.W. Hutmacher, Bone Regeneration Based on Tissue Engineering Conceptions - A 21st Century Perspective, Bone Res. 1 (2013) 216-248. doi:10.4248/BR201303002.

[7] M. Domingos, F. Intranuovo, T. Russo, R. De Santis, A. Gloria, L. Ambrosio, J. Ciurana, P. Bartolo, The first systematic analysis of 3D rapid prototyped poly(??- caprolactone) scaffolds manufactured through BioCell printing: The effect of pore size and geometry on compressive mechanical behaviour and in vitro hMSC viability, Biofabrication. 5 (2013). doi:10.1088/1758-5082/5/4/045004.

[8] F.P.W. Melchels, M.A.N. Domingos, T.J. Klein, J. Malda, P.J. Bartolo, D.W. Hutmacher, Additive manufacturing of tissues and organs, Prog. Polym. Sci. 37 (2012). doi:10.1016/j.progpolymsci.2011.11.007.

[9] N.A. Sears, D.R. Seshadri, P.S. Dhavalikar, E. Cosgriff-Hernandez, A Review of ThreeDimensional Printing in Tissue Engineering, Tissue Eng. Part B Rev. 22 (2016) 298-310. doi:10.1089/ten.teb.2015.0464.

[10] M. Domingos, D. Dinucci, S. Cometa, M. Alderighi, P.J. Bártolo, F. Chiellini, Polycaprolactone Scaffolds Fabricated via Bioextrusion for Tissue Engineering Applications., Int. J. Biomater. 2009 (2009) 239643.

[11] B. Rai, S.H. Teoh, D.W. Hutmacher, T. Cao, K.H. Ho, Novel PCL-based honeycomb scaffolds as drug delivery systems for rhBMP-2, Biomaterials. 26 (2005) 3739-3748. doi:10.1016/j.biomaterials.2004.09.052.

[12] W. Kosorn, M. Sakulsumbat, P. Uppanan, P. Kaewkong, S. Chantaweroad, J. Jitsaard, K. Sitthiseripratip, W. Janvikul, PCL/PHBV blended three dimensional scaffolds fabricated by fused deposition modeling and responses of chondrocytes to the scaffolds, J. Biomed. Mater. Res. - Part B Appl. Biomater. 105 (2017) 1141-1150. doi:10.1002/jbm.b.33658.

[13] M. Domingos, F. Intranuovo, T. Russo, R.D. Santis, A. Gloria, L. Ambrosio, J. Ciurana, P. Bartolo, The first systematic analysis of 3D rapid prototyped poly( $\varepsilon$ - caprolactone) scaffolds manufactured through BioCell printing: The effect of pore size and geometry on compressive mechanical behaviour and in vitro hMSC viability, Biofabrication. 5 (2013). doi:10.1088/1758-5082/5/4/045004. 
[14] G. Caetano, R. Violante, A.B. Sant'Ana, A.B. Murashima, M. Domingos, A. Gibson, P. Bártolo, M.A. Frade, Cellularized versus decellularized scaffolds for bone regeneration, Mater. Lett. 182 (2016) 318-322. doi:10.1016/j.matlet.2016.05.152.

[15] B. Ostrowska, A. Di Luca, L. Moroni, W. Swieszkowski, Influence of internal pore architecture on biological and mechanical properties of three-dimensional fiber deposited scaffolds for bone regeneration, J. Biomed. Mater. Res. - Part A. 104 (2016) 991-1001. doi:10.1002/jbm.a.35637.

[16] L. Moroni, J.R. De Wijn, C.A. Van Blitterswijk, 3D fiber-deposited scaffolds for tissue engineering: Influence of pores geometry and architecture on dynamic mechanical properties, Biomaterials. 27 (2006) 974-985. doi:10.1016/j.biomaterials.2005.07.023.

[17] M. Domingos, F. Chiellini, A. Gloria, L. Ambrosio, P. Bartolo, E. Chiellini, Effect of process parameters on the morphological and mechanical properties of 3D Bioextruded poly $(\varepsilon-$ caprolactone) scaffolds, Rapid Prototyp. J. 18 (2012) 56-67. doi:10.1108/13552541211193502.

[18] J.M. Sobral, S.G. Caridade, R.A. Sousa, J.F. Mano, R.L. Reis, Three-dimensional plotted scaffolds with controlled pore size gradients: Effect of scaffold geometry on mechanical performance and cell seeding efficiency, Acta Biomater. 7 (2011) 1009-1018. doi:10.1016/j.actbio.2010.11.003.

[19] P.J. Ehrlich, L.E. Lanyon, Mechanical strain and bone cell function: A review, Osteoporos. Int. 13 (2002) 688-700. doi:10.1007/s001980200095.

[20] U. Hansen, P. Zioupos, R. Simpson, J.D. Currey, D. Hynd, The Effect of Strain Rate on the Mechanical Properties of Human Cortical Bone, J. Biomech. Eng. 130 (2008) 011011. doi:10.1115/1.2838032.

[21] J.H. McElhaney, Dynamic response of bone and muscle tissue., J. Appl. Physiol. 21 (1966) 1231-1236. doi:10.1152/jappl.1966.21.4.1231.

[22] V.P.W. Shim, L.M. Yang, J.F. Liu, V.S. Lee, Characterisation of the dynamic compressive mechanical properties of cancellous bone from the human cervical spine, Int. J. Impact Eng. 32 (2006) 525-540. doi:10.1016/j.ijimpeng.2005.03.006.

[23] M. Prot, D. Saletti, S. Pattofatto, V. Bousson, S. Laporte, Links between mechanical behavior of cancellous bone and its microstructural properties under dynamic loading, $\mathrm{J}$. Biomech. 48 (2015) 498-503. doi:10.1016/j.jbiomech.2014.12.002.

[24] A. Pilcher, X. Wang, Z. Kaltz, J.G. Garrison, G.L. Niebur, J. Mason, B. Song, M. Cheng, W. Chen, High Strain Rate Testing of Bovine Trabecular Bone, J. Biomech. Eng. 132 (2010) 081012. doi:10.1115/1.4000086. 
[25] M. Prot, T.J. Cloete, D. Saletti, S. Laporte, The behavior of cancellous bone from quasi-static to dynamic strain rates with emphasis on the intermediate regime, J. Biomech. 49 (2016) 1050-1057. doi:10.1016/j.jbiomech.2016.02.021.

[26] E. De Smet, S.V.N. Jaecques, J.J. Jansen, F. Walboomers, J. Vander Sloten, I.E. Naert, Effect of constant strain rate, composed of varying amplitude and frequency, of early loading on peri-implant bone (re)modelling, J. Clin. Periodontol. 34 (2007) 618-624. doi:10.1111/j.1600-051X.2007.01082.x.

[27] G. Caetano, R. Violante, A.B. Sant'Ana, A.B. Murashima, M. Domingos, A. Gibson, P. Bártolo, M.A. Frade, Cellularized versus decellularized scaffolds for bone regeneration, Mater. Lett. 182 (2016) 318-322. doi:10.1016/j.matlet.2016.05.152.

[28] H. Kolsky, An investigation of the mechanical properties of materials at very high rates of loading, Proc. Phys. Soc. Sect. B. 62 (1949) 676-700. doi:10.1088/0370-1301/62/11/302.

[29] J.M. Lifshitz, H. Leber, Data processing in the split Hopkinson pressure bar tests, Int. J. Impact Eng. 15 (1994) 723-733. doi:10.1016/0734-743X(94)90011-9.

[30] W.W. Chen, B. Song, Split Hopkinson (Kolsky) Bar: Design, Testing and Applications., Springer, 2013. doi:10.1007/978-1-4419-7982-7.

[31] W. Chen, F. Lu, B. Zhou, A quartz-crystal-embedded split Hopkinson pressure bar for soft materials, Exp. Mech. 40 (2000) 1-6. doi:10.1007/BF02327540.

[32] B. Song, W. Chen, Split Hopkinson pressure bar techniques for characterizing soft materials, Www.Lajss.Org Lat. Am. J. Solids Struct. 2 (2005) 113-152.

[33] J. Kwon, G. Subhash, Compressive strain rate sensitivity of ballistic gelatin, J. Biomech. 43 (2010) 420-425. doi:10.1016/j.jbiomech.2009.10.008.

[34] A. Benatar, D. Rittel, A.L. Yarin, Theoretical and experimental analysis of longitudinal wave propagation in cylindrical viscoelastic rods, J. Mech. Phys. Solids. 51 (2003) 1413-1431. doi:10.1016/S0022-5096(03)00056-5.

[35] Y. Rotbaum, G. Parvari, Y. Eichen, D. Rittel, Static and Dynamic Large Strain Properties of Methyl Cellulose Hydrogels, Macromolecules. 50 (2017) 4817-4826. doi:10.1021/acs.macromol.7b00270.

[36] E.D.H. Davies, S.C. Hunter, The dynamic compression testing of solids by the method of the split Hopkinson pressure bar, J. Mech. Phys. Solids. 11 (1963) 155-179. doi:10.1016/00225096(63)90050-4.

[37] D. Richler, D. Rittel, On the Testing of the Dynamic Mechanical Properties of Soft Gelatins, Exp. Mech. 54 (2014) 805-815. doi:10.1007/s11340-014-9848-4.

[38] D.J. Frew, M.J. Forrestal, W. Chen, Pulse shaping techniques for testing elastic-plastic 
materials with a split Hopkinson pressure bar, Exp. Mech. 45 (2005) 186-195. doi:10.1177/0014485105052111.

[39] L.. Gibson, M.F. Ashby, Cellular solids structure and properties, Cambridge University Press, 1999.

[40] D.W. Hutmacher, M.E. Hoque, Y.S. Wong, Design, Fabrication and Physical Characterization of Scaffolds Made from Biodegradable Synthetic Polymers in combination with RP Systems based on Melt Extrusion, in: Virtual Prototyp. Bio Manuf. Med. Appl., Springer US, Boston, MA, 2008: pp. 261-291. doi:10.1007/978-0-387-68831-2_12.

[41] S. Yang, K.-F. Leong, Z. Du, C.-K. Chua, The Design of Scaffolds for Use in Tissue Engineering. Part I. Traditional Factors, Tissue Eng. 7 (2001) 679-689. doi:10.1089/107632701753337645.

[42] U. Hansen, P. Zioupos, R. Simpson, J.D. Currey, D. Hynd, The Effect of Strain Rate on the Mechanical Properties of Human Cortical Bone, J. Biomech. Eng. 130 (2008) 011011. doi:10.1115/1.2838032.

[43] D.R. Carter, W.C. Hayes, Bone compressive strength: the influence of density and strain rate., Science. 194 (1976) 1174-6. doi:10.1126/SCIENCE.996549. 


\section{Highlights}

- Quasi static and dynamic mechanical behaviour of bioprinted PCL scaffolds for TE.

- Scaffold's compressive stress is within the range of human trabecular bone.

- Mechanical response at low strain rates $\left(10^{-2}\right)$ is a strong function of porosity.

- 3D printed scaffolds do not exhibit strain rate sensitivity in the range of 1300-2000 $\mathrm{s}^{-1}$

- At the transition from quasi static to dynamic (impact) scaffolds display strain rate sensitivity. 